\title{
Intelligent Vehicle Navigation Path Recognition Model Based on Neural Network
}

\author{
Cheng Chen
}

Shanghai Jian Qiao University, Shanghai,201306, China.

Keywords: neural network; intelligent vehicle; path navigation; fuzzy control; recognition model.

\begin{abstract}
The traditional vehicle navigation path recognition model based on PID realizes the intelligent vehicle path control based on precise mathematical model, which has low robustness and poor intelligent control performance at high speed. Therefore, the design of intelligent vehicle navigation path recognition model based on neural network, intelligent vehicle kinematics model based on neural network, the design of intelligent vehicle navigation path recognition model based on neural network structure, through the vehicle direction control, to achieve control of the intelligent vehicle navigation path. The multilayer feed forward neural network as the basic structure of T-S fuzzy system simulation, implementation of the regulation by training the weights of neural network times, to complete the design of neural network for intelligent vehicle navigation based on path recognition model. The recognition model is trained to reduce the external interference and improve the control accuracy of the recognition model, to realize the control of the intelligent vehicle path navigation. The experimental results show that the designed intelligent vehicle path navigation recognition model based on neural network has high control accuracy and strong robustness, and the intelligent control effect is good.
\end{abstract}

\section{Introduction}

The current intelligent vehicle is the key content of the development of intelligent transportation system, which has high application value in vehicle driving and military and other fields. Intelligent vehicles can reduce the probability of traffic accidents, increase transport efficiency, and promote economic efficiency. The navigation path recognition of intelligent vehicle is the key of the control of intelligent vehicle, which can ensure the stability and fluency of the vehicle autonomous driving, so it has high application value. The traditional vehicle navigation path recognition model based on PID and the intelligent vehicle path control based on accurate mathematical model have low robustness and poor intelligent control performance at high speed. Intelligent vehicle has the quality of high dynamic and time-delay and cannot get accurate mathematical model because of the disturbance of various uncertain factors. The intelligent control technology based on neural network solves the problem existed in PID method well. Fuzzy neural network does not need accurate mathematical model. Based on fuzzy logic, it can complete driver's experience control, and the mapping rules can be generated by training and learning to realize the intelligent control of vehicles. In this paper, an intelligent vehicle navigation path recognition model based on neural network is designed to ensure that intelligent vehicle can track the expected path in the upper cycle with high speed and precision.

\section{An Intelligent Vehicle Navigation Path Recognition Model Based on Neural Network}

\subsection{Intelligent Vehicle Kinematics Model}

This paper provides an analytical basis for the design of the intelligent vehicle navigation path model by tracking the moving path of the intelligent vehicle. Figure 1 is the schematic diagram of the intelligent vehicle path tracking. The position coordinates of the intelligent vehicle are represented by $R(x, y, \theta)^{T},(x, y)$ is the coordinate of the intelligent vehicle in the world coordinate system, and $\theta$ represents the angle between the intelligent vehicle and the $\mathrm{X}$-axis. Point $\mathrm{P}$ denotes the tangent point of the position $\mathrm{R}$ of the intelligent vehicle to the vehicle's moving path, the distance from $\mathrm{R}$ point to $\mathrm{P}$ 
point is expressed by $\mathrm{d}, \theta_{r}$ is the tangential angle at $\mathrm{P}$ point and $\theta_{c}$ is the error angle of direction angle. Therefore, the motion equation of intelligent vehicle is expressed as:

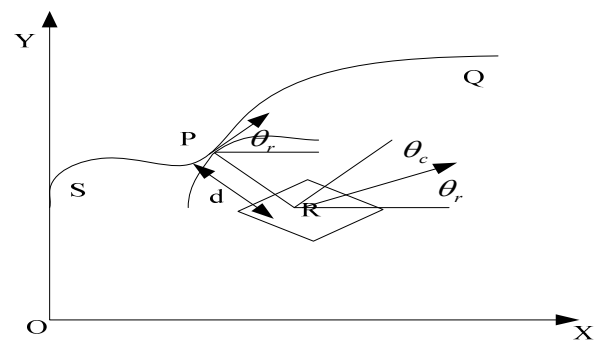

Fig 1. map of intelligent vehicle path tracking

$$
\left\{\begin{array}{l}
\dot{x}=v \cos \theta \\
\dot{y}=v \sin \theta \\
\theta=\omega
\end{array}\right.
$$

In formula $1, \mathrm{v}$ represents the instantaneous linear speed of an intelligent vehicle, $\omega$ represents the angular velocity of an intelligent vehicle. The constraint equation of formula 1 is as follows:

$$
\dot{x} \sin \theta-\dot{y} \cos \theta=0
$$

The position $\mathrm{R}$ of the intelligent vehicle is regarded as the reference material of the moving path of the vehicle, and the current position coordinate of the intelligent vehicle is $R_{c}=\left(x_{c}, y_{c}, \theta_{c}\right)^{T}$

The difference between the directional angle and the reference point angle is:

$$
\theta_{e}=\theta_{r}-\theta_{c} \text {. }
$$

Then the distance between the vehicle position and the reference point is:

$$
d=\sqrt{\left(x_{r}-x_{c}\right)^{2}+\left(y_{r}-y_{c}\right)^{2}}
$$

When the reference point is set on the right side of the intelligent vehicle, the distance between the two points is a positive number $(\mathrm{D}=\mathrm{d})$. If the reference point is on the left side of the vehicle, the distance is expressed as a negative number $(D=-d)$.

\subsection{Structure Design of Intelligent Vehicle Route Navigation Control Model}

In this paper, the intelligent vehicle dynamic permit model based on the above analysis and the structure of the intelligent vehicle path navigation control model based on fuzzy neural network are described in figure 2. After image processing of the path deviation information of the intelligent vehicle, the lateral and azimuth deviation of the intelligent vehicle can be obtained, and then the synthetic deviation can be obtained by merging the path deviation information of the intelligent vehicle. The fuzzy logic is used to control the lateral deviation of the intelligent vehicle. The result of the comprehensive deviation is taken as the input data of the controller, and the front wheel angle is the output result. It can control the driving direction of the vehicle through the neural network and realize the control of the intelligent vehicle path navigation.

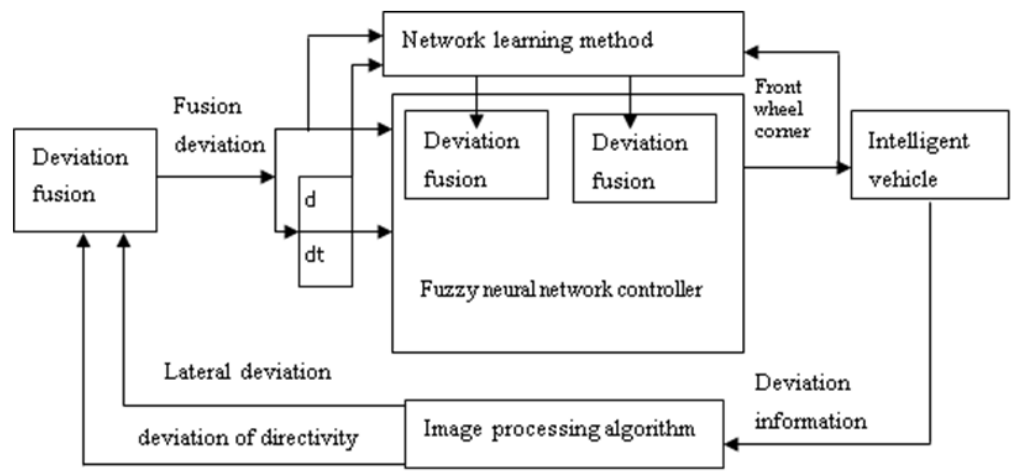

Fig 2. model structure of intelligent vehicle navigation path recognition 


\subsubsection{Design of Intelligent Vehicle Navigation Path Recognition Model Based on Fuzzy Neural Network}

The design principle of intelligent vehicle navigation path recognition model based on fuzzy neural network is as follows: Multi-layer feedforward neural network is used as the basic structure to simulate the T-S fuzzy system, and the weights of the network can be adjusted by training for several times, which can optimize the operation of the network. The combination of fuzzy technology and neural network makes neural network have strong reasoning and association ability. The fuzzy clustering center is obtained from the vehicle data center by the fuzzy C-means clustering method. The fuzzy clustering center is regarded as the former center of the rule. The given data is divided into $\mathrm{n}$ different categories, and $\mathrm{n}$ kinds of clustering centers are represented by $c_{j}=\left(c_{1 j}, c_{2 j}, c_{3 j}\right) \quad j=1,2, \ldots n$; The form of $\mathrm{n}$ fuzzy rules is as follows:

$$
\begin{gathered}
\text { If } x_{1} \text { is } A_{1}^{1}\left(x_{1}\right) \text { and } x_{2} \text { is } A_{2}^{1}\left(x_{2}\right) \\
\text { then } y \text { is } y^{1}, 1=1,2, \ldots, n
\end{gathered}
$$

In this paper, Gao Si function is adopted as the regular pre-function of T-S fuzzy neural network, which makes the function curve smooth. The expression is as follows:

$$
\mu_{i j}\left(x_{i}\right)=\exp \left(-\left(\frac{x_{i}-c_{i j}}{b_{i j}}\right)^{2}\right) i=1,2 ; j=1,2, \ldots, n
$$

The center and width of Gao Si function are denoted by $c_{\mathrm{ij}}$ and $b_{\mathrm{ij}}$ respectively, and the center is the cluster center after fuzzy C- clustering. In this paper, the partition number of input variables is set to 7 , that is, the synthetic deviation is mapped to seven fuzzy subsets, and the resulting fuzzy neural network structure is shown in figure 3. Different levels accomplish different tasks and pass the results of the task to the next level.

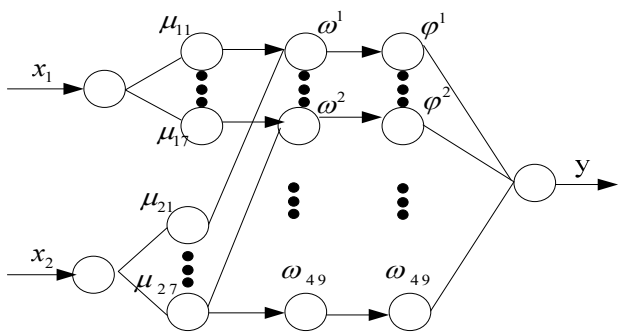

Fig 3. Fuzzy Neural Network

The first layer is the data input layer, which mainly receives the deviation and the rate of deviation change, and its nodes are directly connected with the data, which can normalize the input data, and the processing results are transferred to the next layer to continue processing. The second layer is the generating layer of membership function, which is responsible for the fuzzy operation of the input data and the conversion of the data into fuzzy quantities, that is, the membership degree of the fuzzy subset. The membership function is the Gao Si function after clustering.

$$
\mu_{i j}\left(x_{i}\right)=\exp \left(-\left(\frac{x_{i}-c_{i j}}{b_{i j}}\right)^{2}\right)
$$

In this formula, $\mu_{\mathrm{ij}}$ denotes the $j$ th membership function of the variable $i$, and the data input value of the controller is expressed by $x, c$ represents the center of the function, $b$ is the width of the function. The value of $i$ is 1 or 2 , which represents the dimension of the input variable, $j$ represents the number of neurons.

The third and fourth layers are the fuzzy inference layer, which can optimize the membership function and build the fuzzy rules. The neuron in the third layer is the antecedent part of fuzzy rules, the output result is the weight of the rule:

$$
\omega^{1}=\mu_{A_{1}^{1}}\left(x_{1}\right) \mu_{A_{2}^{1}}\left(x_{2}\right)
$$


The fuzzy set $A_{1}^{1}, A_{2}^{1}$ and $\mu_{A_{1}^{1}}\left(x_{1}\right)$ in the first rule in the above expression mean that the input $\mathrm{x}$ in the first rule belongs to the membership of the $\mu$-set $A_{1}^{1}$. In the fourth layer of the normalization process, the output of each neuron represents the proportion of the corresponding rule after the normalization operation.

$$
\varphi^{l}=\frac{\omega^{l}}{\sum_{m=1}^{n} \omega^{m}} \quad l=1,2, \ldots, n
$$

The fifth layer represents the output layer of the fuzzy neural network, which controls the output results. The output result is the product of the proportion of the fourth layer corresponding rule after normalized operation and the weight of the neuron. The expression of the neuron weight is as follows:

$$
\beta^{l}=k^{l} \bullet A=p_{0}^{l}+p_{1}^{l} x_{1}+p_{2}^{l} x_{2}
$$

In the formula, $k^{l}=\left[p_{0}^{l} p_{1}^{l} p_{2}^{l}\right]$ is the parameter in the fuzzy rule, $A=\left[1 x_{1} x_{2}\right], \beta^{l}$ is the latter part of the fuzzy rule.

If $\mathrm{n}$ control rules are used altogether, the output results of the fifth layer, that is, the final navigation path control results of the intelligent vehicle, are as follows:

$$
y=\sum_{l=1}^{n} \beta^{1} \varphi^{l}
$$

The fuzzy neural network vehicle controller can form 49 fuzzy rules through the fuzzy C-mean classification and the fuzzy neural network. If the first rule is set to $\mathrm{R}$, the expression of the rule is:

$$
\begin{gathered}
R^{l}: \text { if } x_{1} \text { is } A_{1}^{1} \text { and } x_{2} \text { is } A_{2}^{1}, \text { then } \\
u_{l}=p_{0}^{l}+p_{1}^{l} x_{1}+p_{2}^{l} x_{2}(l=1,2, \ldots, 49)
\end{gathered}
$$

In the formula, $u_{l}$ denotes the output of rule $\mathrm{i}$.

\subsubsection{The Training of Fuzzy Neural Network Intelligent Vehicle Navigation Path Recognition Model}

From the structure of the fuzzy neural network, we can see that the first three including input layers, hidden layers and output layers of the intelligent vehicle navigation path recognition model constructs a forward network structure, and the fourth layer mainly carries on the neuronal reasoning. Drivers drive smart vehicles to collect road traffic data. During the vehicle's travel, drivers collect $l_{e}, U$ and $v_{0}$ values every $60 \mathrm{~ms}$ and collect the results into a single data file. The data collection time is 20 minutes in total. The navigation path of driving intelligent vehicle includes four paths: straight line-curve-straight line or S curve-straight line-S curve. Abnormal data were removed and a neural network based on intelligent vehicle navigation path recognition model was obtained. After driving many times, 600 sets of data of the path $S$ curve- straight $-S$ curve were obtained, and the initial learning rate of the controller is 0.3 , the momentum coefficient is 0.9 , and the error probability is $0.1 \%$. The weights $\mathrm{W} 1$ and threshold $\mathrm{B}_{1}$ of the hidden layer and the $\mathrm{W}_{2}$ and $\mathrm{B}_{2}$ of the output layer are obtained after several training. The learning efficiency of the intelligent vehicle navigation path recognition model using TS model and fuzzy neural network design is $5 \%$. The training sample data is imported into the neural network structure for offline training. After training, the training sample has a memory function, and offline training can be adjusted the fuzzy parameter on the training sample, it makes the fuzzy neural network effectively simulate the relationship between input and output, and reduces external interference, and improves the control accuracy of the intelligent vehicle navigation path recognition model.

\section{Experimental Results and Analysis}

In the experiment, the neural network-based intelligent vehicle navigation path recognition model is used to control a certain type of intelligent vehicle. The recognition model performs linear path and circular path navigation for smart vehicles at a speed of $1 \mathrm{~m} / \mathrm{s}$, and then implements MATLAB 
simulation, the original sample data was obtained from driving a smart vehicle. It is assumed that the data tracked in the experiment can collect accurate data in 20 samples, the upper angular velocity in the linear motion is 0 degrees per second, and the angular velocity in the circular shape is 12.264 degrees per second. Twenty samples were simulated and trained 1,000 times. Table 1 and Table 2 are the navigation and tracking data of the recognizing model on the linear and circular path of the experimental intelligent vehicle.

Tab 1. path navigation and tracking data control table (straight line)

\begin{tabular}{|c|c|c|c|c|c|c|c|}
\hline \multirow{2}{*}{\multicolumn{2}{|c|}{ Sample sequence number }} & \multicolumn{3}{|c|}{ actual value } & \multicolumn{3}{|c|}{ actual value } \\
\hline & & $\theta$ & $\mathrm{D}$ & $\omega$ & $\theta$ & $\mathrm{D}$ & $\omega$ \\
\hline \multicolumn{2}{|c|}{1} & 44.1 & -3.77 & 21.1 & 46.11 & -4.77 & 18.83 \\
\hline \multicolumn{2}{|c|}{2} & 24.1 & -4.68 & 23.1 & 26.16 & -5.68 & 21.17 \\
\hline \multicolumn{2}{|c|}{3} & 3.1 & -4.12 & 21.1 & 4.65 & -6.12 & 20.11 \\
\hline \multicolumn{2}{|c|}{4} & -16.1 & -5.16 & 18.1 & -16.22 & -6.17 & 16.79 \\
\hline \multicolumn{2}{|c|}{5} & -34.1 & -4.66 & 6.6 & -34.24 & -5.69 & 6.47 \\
\hline \multicolumn{2}{|c|}{6} & -41.6 & -4.31 & -6.1 & -41.61 & -5.32 & -5.57 \\
\hline \multicolumn{2}{|c|}{7} & -34.6 & -3.63 & -1.1 & -34.13 & -4.42 & -2.23 \\
\hline \multicolumn{2}{|c|}{8} & -33.6 & -2.83 & -6.6 & -32.68 & -2.83 & -5.71 \\
\hline \multicolumn{2}{|c|}{$\ldots$} & $\ldots$ & $\ldots$ & $\ldots$ & $\ldots$ & $\ldots$ & $\ldots$ \\
\hline \multicolumn{8}{|c|}{ Tab 2. path navigation and tracking data control table (circular) } \\
\hline \multirow{2}{*}{ number } & \multicolumn{3}{|c|}{ actual value } & \multicolumn{4}{|c|}{ actual value } \\
\hline & $\theta$ & $\mathrm{D}$ & $\omega$ & $\theta$ & & $\mathrm{D}$ & $\omega$ \\
\hline 1 & 0 & -4.56 & 20.31 & 0 & & -4.25 & 22.26 \\
\hline 2 & -8.22 & -4.15 & 21.3 & -10.36 & & -4.16 & 21.33 \\
\hline 3 & -24.31 & -4.56 & 12.34 & -23.45 & & -4.28 & 13.26 \\
\hline 4 & -29.66 & -4.32 & 12.36 & -30.26 & & -4.68 & 9.23 \\
\hline 5 & -34.82 & -3.75 & 6.1 & -34.26 & & -3.26 & 6.21 \\
\hline 6 & -35.17 & -3.65 & 6.1 & -35.36 & & -3.46 & 5.23 \\
\hline 7 & -35.33 & -2.53 & 5.1 & -36.12 & & -2.56 & 5.26 \\
\hline 8 & -33.62 & -2.45 & 5.1 & -36.15 & & -2.15 & 5.12 \\
\hline$\ldots$ & $\ldots$ & $\ldots$ & $\ldots$ & $\ldots$ & & $\ldots$ & $\ldots$ \\
\hline
\end{tabular}

In the above two tables, $\theta$ indicates the direction angle error, $\mathrm{D}$ indicates the distance error, and $\omega$ indicates the angular velocity error. From the tracking results of straight line and circular path in Table 1 and Table 2, the vehicle angular velocity becomes more stable, the convergence is higher and the robustness is stronger after the fuzzy neural network training of the model in this paper.

Fig. 4 shows the motion trajectory error in the intelligent vehicle route guidance after the control of this recognition model. It can be seen from the figure that the error of the motion trajectory is within 0.5 meters, which indicates that the accuracy of the model is higher and the intelligent vehicle path navigation can be accurately identified. It also can be seen from the figure that the peaks with higher fluctuations are in the locations where the road curvature is high and the relative volatility is high, which is in line with the actual driving experience. 


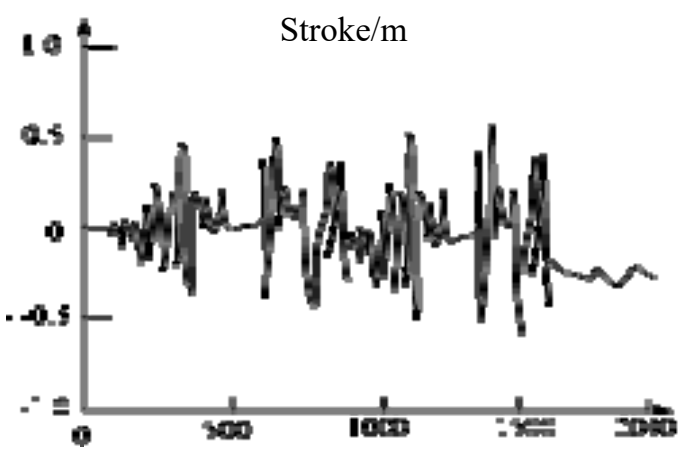

Fig 4. trajectory error of intelligent vehicle

\section{Conclusion}

This design is an intelligent vehicle navigation path recognition model based on neural network. It realizes the experience control of intelligent vehicle path through fuzzy neural network and improves the control precision of the model by training the recognition model and realizes the high precision control of intelligent vehicle path.

\section{References}

[1]. Ni Jie, Liu Zhiqiang. The model of lane change based on driver decision-making mechanism [J]. Transportation System Engineering and Information, 2016, 16 (1): 58-63.

[2]. Zhou Qing, Zou van. Conflict identification model of industrial technology innovation strategy alliance based on neural network [J]. Technology Economy, 2016, 35 (4): 44-51.

[3]. Liu Zhanwen, Zhao Xiangmo, Li Qiang, et al. Method of identification of traffic signs based on graph model and convolution neural network [J]. Journal of Transport Engineering, 2016, 16 (5): 122-131.

[4]. Should be jujuan, float clean, Zhang Li Xiang. Research on the reverse model identification of magnetorheological dampers based on neural network [J]. Computer Simulation, 2015, 32 (12): 408-412.

[5]. Deng Zhaoxue, Zheng Ling, Guo Minmin, et al. Identification of magnetorheological suspension model based on genetic BP neural network [J]. Journal of Electronic Science and Technology, 2014, 43 (6): 955-960.

[6]. Meng Xianghai, Qin Wei, Deng Xiaoqing. Highway accident prediction model in mountain ridge area based on neural network [J]. Highway Traffic Technology, 2016, 33 (3): 102-108.

[7]. Lin Wenru, Chen Tenglin, Lin Guofu. Vehicle route optimization model based on improvement neural network [J]. Computer Engineering and application, 2015, 51 (16): 239-242.

[8]. Zhuyun, Gao Ningbo, Jung Ryeowon. A comprehensive measurement model of road traffic safety based on neural networks [J]. China Journal of Security Science, 2015, 25 (10): 22-28.

[9]. Jiang Shizheng, Xu Rong, Chen Qi Mei. Short-term traffic flow prediction for complex road network based on variable selection neural network [J]. Journal of Shanghai Jiao Tong University, 2015, 49 (2): 281-286.

[10]. Zhang Meng, Lou Shengli, Ding Liang. The driver's interpersonal and emotional risk model based on back-propagation neural network [J]. Highway Traffic Technology, 2015, 32 (1): $140-144$. 